\title{
Force-mediated parametric generation in nano-optomechanical structures
}

\author{
Q. Lin, J. Rosenberg, D. Chang, and O. Painter \\ Department of Applied Physics, California Institute of Technology, Pasadena, CA 91125 \\ linq@caltech.edu
}

\begin{abstract}
We report a novel scheme for freely engineering phase matching, enabling efficient parametric generation at arbitrary frequencies in mechanically compliant nano-optomechanical structures. (C)2010 Optical Society of America

OCIS codes: $130.3990,190.4390$
\end{abstract}

With their great potential for generating both classical new light and photonic quantum states, optical parametric processes through either $\chi^{(2)}$ or $\chi^{(3)}$ nonlinearity have attracted long-lasting interest since the invention of lasers up to recent attempts to their excitations in micro/nanocavities [1-12]. Unfortunately, the stringent requirement for phase matching imposes a serious challenge on the efficient utilization of these processes and there has been a long history of struggle for this even since the first observation of second-harmonic generation [1]. Various approaches have been proposed to mitigate this difficulty in micro/nanocavities [2-12]. However, only very limited attempts have succeeded along this line, all requiring a tremendous effort in engineering the cavity dispersion [3,5,7,11]. Here we report a novel scheme which can ultimately solve this problem, enabling efficient parametric generation at arbitrary frequencies.

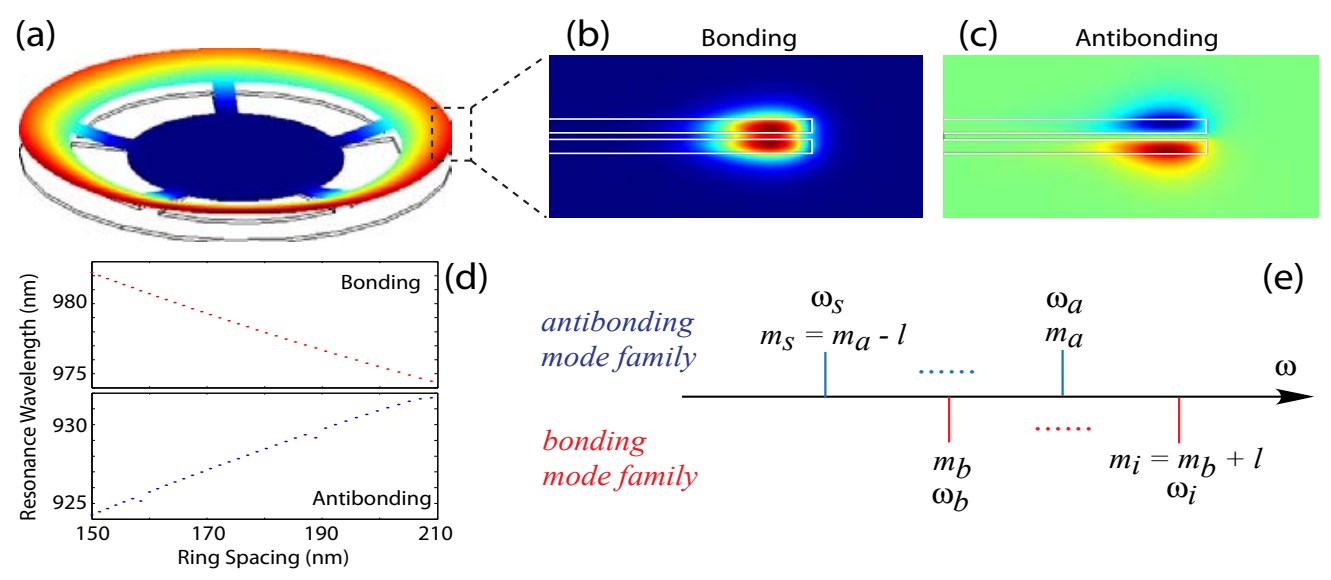

Figure 1: (a) Schematic of the "spiderweb" NOMS. (b) and (c) Simulated field profiles for the cavity supermodes. (d) The cavity resonance as a function of ring spacing. (e) Schematic of momentum and energy conservation among the four cavity modes involved in four-photon scattering.

Our proposed scheme is based upon the force-induced cavity tuning in nano-optomechanical structures (NOMS) [13]. As illustrated in Fig. 1, the strong coupling between two closely spaced microresonators splits the cavity modes into a symmetric and anti-symmetric supermode (Fig. $1 \mathrm{~b}$ and c), resulting in a gradient force which is attractive and repulsive for the two modes, respectively. As the photon energy is lowered (lifted up) for the bonding (antibonding) mode, the cavity resonances of the two modes are tuned in opposite directions in spectrum when the resonator gap is changed (Fig. 1d). Note that this effect is uniform for the entire family of the bonding (or antibonding) modes, as shown clearly in Fig. 2 for a mechanically compliant "spiderweb" resonator. By launching optical power into a bonding mode at the telecom band, the induced optical force strongly attracts the two microrings towards each other. As a result, in the 980-nm band, the bonding mode family moves towards red while the anti-bonding mode family moves towards blue, resulting in a significant change in the frequency spacing between the bonding and antibonding modes. As this frequency separation can be freely controlled through optically actuating the resonator spacing, it readily provides a convenient and efficient approach for engineering the phase matching if the bonding and anti-bonding modes are combined for exciting parametric generation. Here we use four-wave mixing (four-photon scattering) as an example to illustrate the power of this scheme. The same idea can be readily applied to other parametric processes.

Four-wave mixing requires simultaneous conservation of momentum and energy among the four interacting photons. Assume that we launch optical power into a bonding and anti-bonding mode with mode numbers of $m_{b}$ and 


\section{QTuA1.pdf}

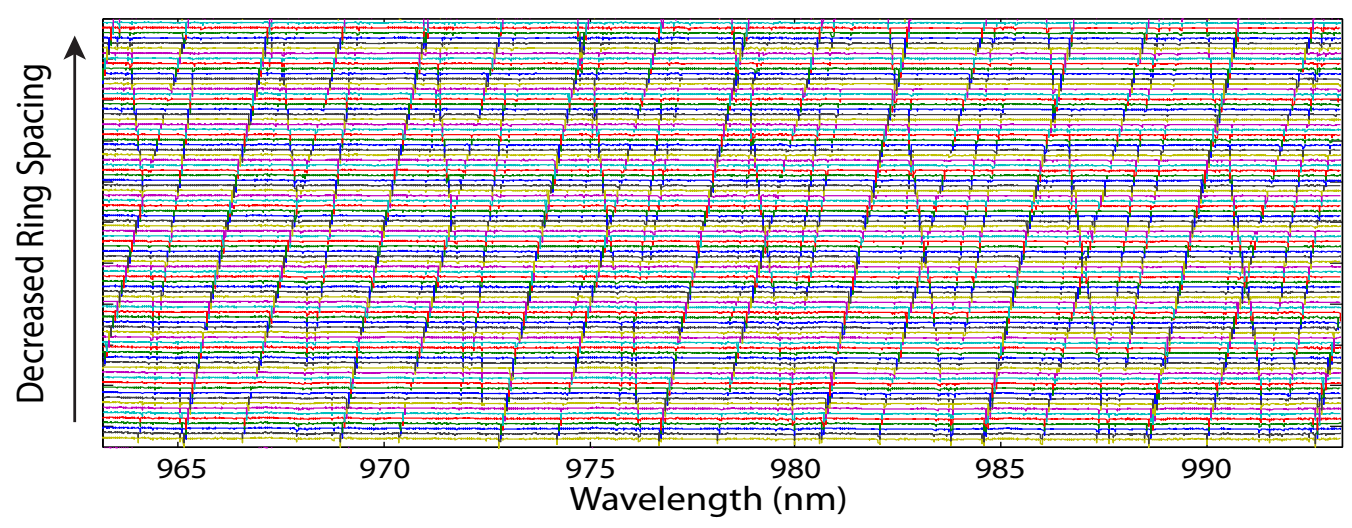

Figure 2: Recorded cavity transmission spectra as a function of ring spacing, clearly showing the opposite tuning of the bonding and antibonding mode family. The "spiderweb" NOMS consists of two 400-nm thick silica rings separated by a 150-nm gap, with a diameter of $60 \mu \mathrm{m}$.

$m_{a}$ and corresponding frequencies of $\omega_{b}$ and $\omega_{a}$, respectively (Fig. 1e). For any signal mode at the Stokes side with a mode number of $m_{s}=m_{a}-l$ and a frequency of $\omega_{s}$, there is always an idler mode at the anti-Stokes side with a mode number of $m_{i}=m_{b}+l$ (frequency at $\omega_{i}$ ) which satisfies the momentum conservation for the four-photon scattering: $m_{b}+m_{a}=m_{s}+m_{i}$ (Fig. 1e). More importantly, by actuating the resonator gap, the frequency spacing $\omega_{b}-\omega_{s}$ can be easily engineered to match $\omega_{i}-\omega_{a}$, leading to the energy conservation for four-photon scattering: $\hbar \omega_{b}+\hbar \omega_{a}=\hbar \omega_{s}+\hbar \omega_{i}$. Note that, in principle, this process can be realized for arbitrary frequency spacing between the two bonding modes $\omega_{a}-\omega_{s}$ (or between the two anti-bonding modes $\omega_{b}-\omega_{i}$ ), as long as the cavity resonance can be tuned by one free spectral range, a capability readily available in our device [13]. Therefore, this powerful technique is able to excite parametric generation anywhere in the spectrum.

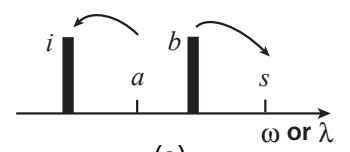

(a)

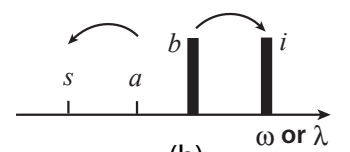

(b)

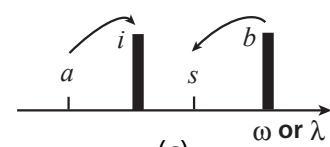

(c)

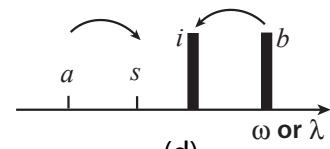

(d)

Figure 3: Schematic for single-photon state transfer in four possible configurations, where $a$ is the input single photon, $s$ is generated single photon, $i$ and $b$ are assisting pump modes.

Apparently, the proposed scheme would be quite useful for generating new light tunable over a broad bandwidth. Apart from this popular classical application, such freely-controllable parametric generation is particularly efficient in single-photon state transfer. Figure 3 shows four possible configurations for this purpose. Detailed analysis shows that these systems are governed by a Hamiltonian of the form $\mathscr{H}=-\hbar g_{2} a_{s}^{\dagger} a_{a}+$ h.c., with $g_{2}=\frac{4 \hbar c \omega_{0}^{2} n_{2}}{n_{0}^{2} V_{\text {eff }}} \sqrt{N_{b} N_{i}}$ where $n_{2}$ is the Kerr nonlinear coefficient, $V_{\text {eff }}$ is the effective mode volume, and $N_{b}$ and $N_{i}$ are the intracavity photon numbers for the assisting mode $b$ and $i$. Detailed numerical analysis shows that even moderate photon numbers $N_{b}$ and $N_{i}$ can provide nearly complete transfer of the single-photon state, with an efficiency much higher than other schemes [12]. This method shows great potential for applications in future quantum information processing on chip.

\section{References}

[1] R. W. Boyd, Nonlinear Optics, $2^{\text {nd }}$ Ed. (Academic Press, New York, 2003).

[2] C. Conti, et al, "Optical parametric oscillations in isotropic photonic crystals," Opt. Express 12, 823 (2004).

[3] P. DelHaye, et al, "Optical frequency comb generation from a monolithic microresonator," Nature 450, 1214 (2007).

[4] Z. Yang, et al, "Enhanced second-harmonic generation in AlGaAs microring resonators," Opt. Lett. 32, 826 (2007).

[5] T. Carmon, et al, "Visible continuous emission from a silicamicrophotonic device by third-harmonic generation," Nature Phys. 3, 430 (2007).

[6] Y. Xu, et al, "Second Order Parametric Processes in Nonlinear Silica Microspheres," Phys. Rev. Lett. 100, 163905 (2008).

[7] A. A. Savchenkov, et al, “Tunable Optical Frequency Comb with a Crystalline WGM Resonator," Phys. Rev. Lett. 101, 093902 (2008).

[8] J. B. Khurgin, et al, "Suspended AlGaAs waveguides for tunable difference frequency generation in mid-infrared,", Opt. Lett. 33, 2904 (2008).

[9] A. Andronico, et al, "Difference frequency generation in GaAs microdisks," Opt. Lett. 33, 2026 (2008).

[10] A. Melloni, et al, "Four-wave mixing and wavelength conversion in coupled-resonator optical waveguides," JOSA B 25, C87 (2008).

[11] J. S. Levy, et al, "Monolithically integrated multiple wavelength oscillator on silicon," arXiv:0907.1077.

[12] M. W. McCutcheon, et al, "Broad-band spectral control of single photon sources using a nonlinear photonic crystal cavity," arXiv:0903.4706.

[13] J. Rosenberg, et al, "Static and dynamic wavelength routing via the gradient optical force," Nature Photonics 3, 478 (2009). 